\title{
Odontología Forense III: Rugas Palatinas y Huellas Labiales en Identificación Forense
}

\author{
Forensic Dentistry III: Palatal Rugae and Lip Prints in Forensic Identification
}

\author{
Gabriel M. Fonseca, ${ }^{* * *}$; Mario Cantín ${ }^{* * * * *}$ \& Joaquín Lucena ${ }^{*+* *, * k+* *}$
}

FONSECA, G. M.; CANTÍN, M. \& LUCENA, J. Odontología Forense III: Rugas palatinas y huellas labiales en identificación forense. Int. J. Odontostomat., 8(1):29-40, 2014.

RESUMEN: Aunque existen métodos de identificación humana más o menos frecuentes en uso, todos ellos se soportan en la comparación inequívoca, científica y fiable, de una información conocida con la obtenida de los restos o de las huellas a procesar. Desde una perspectiva odontológica forense, los surcos labiales, las rugas palatinas y sus negativos o huellas, han sido reconocidos como patrones morfológicos que permitirían identificar a una persona en circunstancias específicas. Estos elementos han sido estudiados tanto comparativa como independientemente buscando detectar las características individuales y poblacionales que permitan reforzarlos como herramientas significativas: se ha informado que tanto la Queiloscopía (el estudio de las huellas labiales) como la Rugoscopía (el estudio de las rugas palatinas) definen rasgos únicos para cada individuo, por lo tanto utilizables razonablemente para una identificación forense. Dado que los reportes de casos forenses permiten identificar cambios en las conductas criminales, en los patrones morfológicos característicos o en la manera de presentar la evidencia pericial, se presenta una revisión de reportes de casos con utilización de evidencia rugoscópica y queiloscópica, junto con discutir sus proyecciones hacia la comunidad científica forense.

PALABRAS CLAVE: odontología forense, rugoscopía, queiloscopía, reportes de casos.

\section{INTRODUCCIÓN}

La identificación humana es uno de los campos más abordados e investigados por los científicos forenses y, sea para establecer la identidad de restos humanos en diferentes grados de vulneración o la de sospechosos de haber cometido un crimen, el proceso requiere de una combinación de recursos correctamente aplicados, cuyos resultados deben ser apropiadamente interpretados y presentados al proceso judicial. Aunque existen métodos más o menos frecuentes en su uso para este procedimiento, todos ellos se soportan en la comparación inequívoca, científica y fiable, de una información conocida con la obtenida de los restos o de las huellas a procesar. La cavidad oral ha probado ser fuente de datos y posibilidades con la que este proceso podría realizarse en una cohorte definida. Entre ellos, los surcos labiales, las rugas palatinas y sus negativos o huellas, han sido reconocidos como patrones morfológicos que permiti- rían identificar a una persona en circunstancias específicas. Estos elementos han sido estudiados tanto comparativa como independientemente buscando detectar las características individuales y poblacionales que permitan reforzarlos como herramientas significativas: se ha informado que tanto la Queiloscopía (el estudio de las huellas labiales) como la Rugoscopía (el estudio de las rugas palatinas) definen rasgos únicos para cada individuo, por lo tanto utilizables razonablemente para una identificación forense (Caldas et al., 2007).

Dado que los reportes de casos en ciencias forenses permiten identificar cambios en las conductas criminales, en los patrones morfológicos característicos o en la manera de presentar la evidencia pericial, y con ello formular hipótesis, diseñar estudios sistemáticos prospectivos o simplemente ayudar a la

* Profesor Titular, Cátedra de Anatomía Patológica B, Facultad de Odontología, Universidad Nacional de Córdoba, Córdoba, Argentina.

* CIMA, Departamento Odontología Integral Adultos, Facultad de Odontología, Universidad de La Frontera, Temuco, Chile.

*** Centro de Investigación en Ciencias Biomédicas, Universidad Autónoma de Chile, Temuco, Chile.

**** Profesor Asociado, Departamento de Anatomía Patológica y Medicina Legal, Universidad de Cádiz, Cádiz, España.

**t*** Servicio de Patología Forense, Instituto de Medicina Legal, Sevilla, España. 
resolución de un proceso judicial (Madea, 2007), se presenta una revisión de reportes de casos con utilización de evidencia rugoscópica y queiloscópica, y se discuten sus proyecciones hacia la comunidad científica forense.

\section{RUGOSCOPÍA}

Rugoscopía, término acuñado por el investigador español Trobo Hermosa, es el nombre que se da al estudio de las rugas palatinas para establecer la identidad de un individuo. Sumado a sus defendidas características de unicidad, individualidad, perennidad e inmutabilidad, la posición interna en la cavidad oral rodeada por dientes (en ocasiones), lengua y mejillas, las situaría en una ubicación protegida a los traumatismos y al fuego a diferencia de las huellas dactilares que pueden verse fácilmente destruidas por ambos (Caldas et al.). Aunque existe una robusta y constante referencia a sus citas históricas, tipos de clasificación y a trabajos originales de valoración poblacional, en esta revisión solo fueron encontrados tres casos de identificación mediante este tipo de evidencia.

\section{Desafortunadamente infrecuente.}

El cuerpo severamente carbonizado de una mujer edéntula residente de la pequeña villa de McGregor (Sudáfrica), fue encontrado con un juego de prótesis completas alojadas en su cavidad oral. La prótesis maxilar se encontraba fracturada longitudinalmente y sólo el fragmento más grande estaba disponible para examen; la prótesis mandibular estaba intacta. Al ser hallado otro juego de prótesis en la casa de la supuesta víctima, se realizaron modelos de yeso de ambas prótesis maxilares. Las rugas y el rafe fueron delineados y fotografiados en proximidad comparativa, y con ayuda de láminas de acetato se realizaron las superposiciones para poner en evidencia cualquier tipo de concordancia. Similar procedimiento se realizó con los rebordes alveolares maxilares y mandibulares en los cuatro modelos. La positividad en la identificación fue asignada en forma inmediata y segura (Fig. 1). Este caso fue publicado por Thomas \& van Wyk (1988), odontólogos de la Universidad de Stellenbosch en Sudáfrica, quienes destacaron la no existencia de reportes previos sobre este tipo de identificación. Los autores consideraron que la calidad de impresión de las rugas palatinas (por ende del cotejo identificatorio) va a depender de la edad del individuo, condición de las prótesis, capacidad y experiencia del observador. Sobre el caso en particular (referencia omnipresente en toda publica-

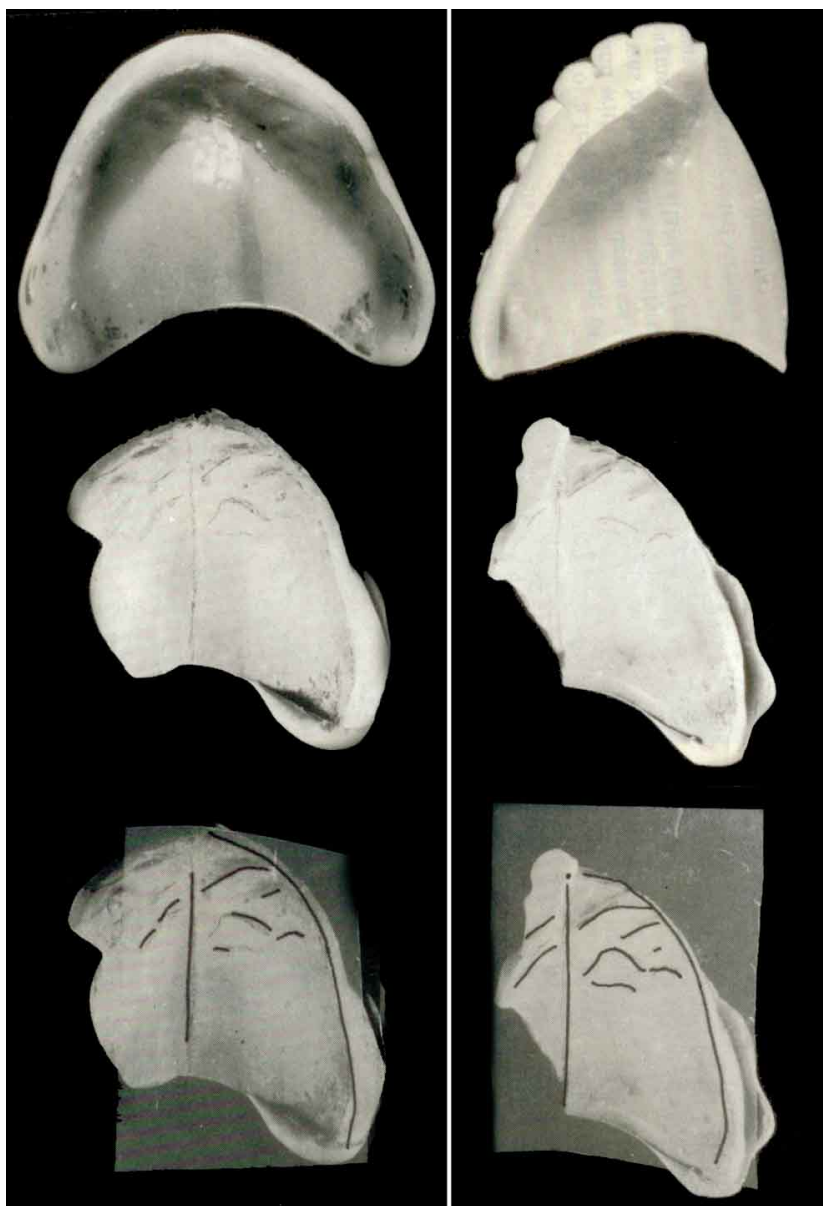

Fig. 1. Caso reportado por Thomas \& van Wyk de identificación por cotejo antemortem-postmortem con ayuda de rugas palatinas revenidas de prótesis maxilares. Columna de la izquierda: Procedimientos sobre prótesis maxilar provista por los familiares de la víctima. Columna de la derecha: Procedimientos sobre prótesis maxilar obtenida de boca de la víctima. Arriba: Prótesis; Centro: Escayolas (modelo de yeso); Abajo: Transparencia de cotejo (Adaptado de Thomas \& van Wyk, 1988).

ción sobre el tópico), los autores destacaron la "desafortunadamente infrecuente" ayuda de contar con otro juego de prótesis pertenecientes a la mujer fallecida.

\section{Desafortunadamente infrecuente II.}

El Instituto Nacional de Medicina Legal y Ciencias Forenses de Colombia (Regional Oriente), reportó el hallazgo en 1993 de un cadáver de sexo femenino no identificado en la población de Cundinamarca (Colombia). El cuerpo estaba esqueletizado pero aún con tejidos blandos en el paladar. Familiares de una desaparecida hace 15 días aportaron una prótesis dental que se le estaba realizando, de la que se obtu- 
vo un modelo de yeso para compararlo con otro obtenido del paladar de la víctima. Esto permitió cotejar la disposición, forma y tamaño de las rugas palatinas logrando una identificación positiva de la mujer. Los autores refieren que no pudo obtenerse una historia clínica odontológica antemortem (AM), y que solo la oportuna provisión de la prótesis por los familiares permitió una comparación respaldada por parámetros válidos identificatorios con carácter biométrico (Aparicio Castellanos et al., 2007).

\section{El accidente aéreo.}

El 15 de Septiembre de 1995, un avión Fokker 50 de Malaysian Airlines se precipitó en la periferia del aeropuerto de Tawau (Malasia), y se consumió en llamas carbonizando a 34 víctimas, de las que 7 fueron exclusivamente identificadas por odontología. Una en particular, logró ser identificada por la comparación de las rugas palatinas de su prótesis superior con una de repuesto que facilitaron los familiares. El caso fue reportado por Nambiar et al. (1997), quienes mencionan dificultades para obtener y descifrar la información de las fichas dentales y que la inclusión de marcas o números de serie en las prótesis podría favorecer el proceso de identificación.

\section{QUEILOSCOPÍA}

Se ha definido a la Queiloscopía (del Griego cheilos, labios, y skopein, exploración), como el estudio de los patrones morfológicos de los surcos labiales y las huellas que estos dejan. Si bien la literatura ofrece una variada y abundante cantidad de trabajos sugiriendo clasificaciones, aplicaciones de corte antropológico y métodos de recolección, análisis e interpretación estos patrones (Caldas et al.; Dineshshankar et al., 2013), los reportes de casos en los que la queiloscopía ha sido usada con éxito no guardan una relación proporcional con estos estudios.

\section{La huella en el parachoques.}

Sin duda uno de los casos más citados por la literatura lo constituye el que hace referencia a la identificación de un criminal mediante huellas labiales en una investigación bastante particular: Casos de atropello y fuga en el que una joven sufrió importantes lesiones en su espalda. El automóvil sospechoso fue localizado y la relación con la víctima se pudo establecer por una huella labial encontrada por los equipos de investigación en el parachoques del automóvil. Esta huella sería luego cotejada positivamente con los la- bios de la víctima (quien había sobrevivido al atropeIlo), específicamente por 17 puntos coincidentes. El caso es referido por el prolífico Director Medicolegal de la Policía del Estado de Michigan, Dr. LeMoyne Snyder, en la primera edición revisada de su libro "Homicide Investigation: Practical Information for Coroners, Police Officers, and Other Investigators" (Snyder, 1950) y en las sucesivas reediciones. Este reporte es casi una cita obligada en la literatura más relevante y actual dedicada a la queiloscopía (Caldas et al.; Dineshshankar et al.). Sin embargo, al consultar las fuentes primarias resulta curiosa la ausencia del caso en la primera edición de la obra (1944) y luego, en las posteriores ediciones, la leyenda "cortesía de Leland V. Jones, Los Angeles" junto al reporte.

Leland V. Jones, Profesor de la Universidad del Sur de California y Teniente del Departamento de Policía de Los Angeles, es reconocido como uno de los pioneros de la investigación criminalística. Sus más de 30 años en la dirección de la División de Investigación Científica y su experiencia de laboratorio forense en unos 10.000 casos con unas 2500 declaraciones como perito, han quedado plasmados en su obra"Scientific Investigation and Physical Evidence. A Handbook for Investigators" (Jones, 1959). Es precisamente en su página 145 donde el caso mencionado se describe en toda su magnitud: "La huella del pintalabios fue descubierta, levantada con cinta de celofán, colocada en fondo blanco y fotografiada. Esas fotografías fueron comparadas con huellas labiales muestreadas de los labios de la chica, y diecisiete puntos de identificación fueron encontrados" (Fig. 2). Dato curioso es que mientras Jones afirma que la huella labial fue hallada en el lado derecho del parachoques (un Dodge coupé según reportes de la época), Snyder hace lo propio mencionando el hallazgo en el lado izquierdo del mismo.

\section{Los casos japoneses}

En Septiembre de 1975, dos casos resueltos con huellas labiales eran presentados en el Séptimo Encuentro Internacional de Ciencias Forenses en Zúrich (Suiza). En el primero, una carta anónima señalada como "confidencial" fue enviada en 1970 a la Oficina del Director General de la Policía Metropolitana de Tokio. Alertaba sobre un artefacto explosivo colocado en los cuarteles de la Policía, y el dorso del sobre ofrecía dos huellas excelentemente marcadas. Las pericias queiloscópicas demostraron positivamente que las huellas no pertenecían a los dos individuos arrestados. En el segundo, huellas labiales halladas en las prendas íntimas de una mujer permitieron eli- 


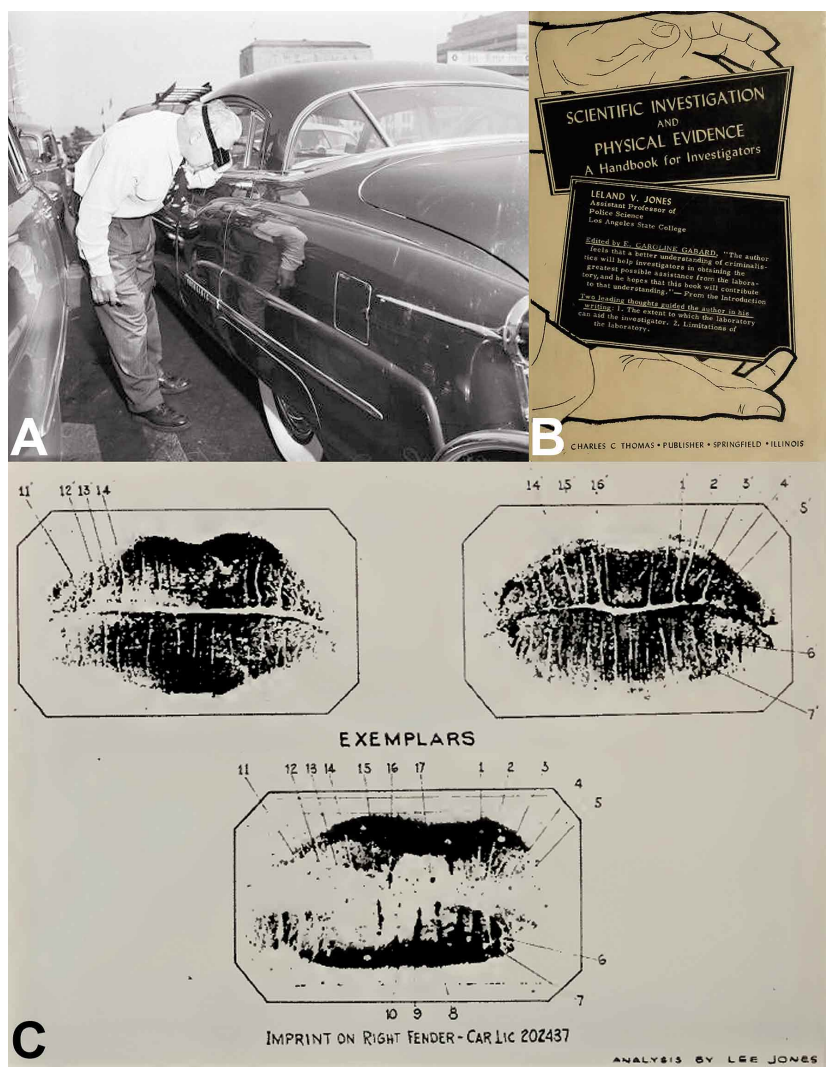

Fig. 2. A. El Teniente del Departamento de Policía de Los Angeles, Prof. Leland "Lee" V. Jones, buscando manchas de sangre en la investigación de un asesinato en Hollywood (Tomado de http://digitallibrary.usc.edu). B. Portada de Scientific Investigation and Physical Evidence. A Handbook for Investigators (Jones, 1959). C. Arriba: ejemplares de huellas labiales tomados de la víctima del atropello y fuga, en el hospital; Abajo: Huella labial levantada con cinta de celofán y colocada en fondo blanco para su fotografía. Nótese en el sector inferior las leyendas "Impresión en el lado derecho del parachoques - Matrícula del automóvil 202437", y "Análisis realizado por Lee Jones" (Jones, 1959).

minar a un sospechoso de un robo e identificar a otro, quien resultó ser su hermano. Las huellas fueron estudiadas con la ayuda de pruebas colorimétricas y radiación UV (Suzuki \& Tsuchihashi, 1975). Los casos serían luego publicados por los Dres. Kazuo Suzuki y Yasuo Tsuchihashi de la Facultad de Odontología de Tokio, en la revista Acta Criminologica Japonica configurando uno de los artículos más referenciados en la temática. No serían los únicos casos conocidos de estos investigadores: la huella labial en una carta enviada al presidente de los Ferrocarriles Nacionales de Japón permitió que varios pasajeros fueran puestos bajo vigilancia; un pañuelo con una impresión labial favoreció la identificación del culpable de la tortura y asesinato de una mujer japonesa (Kandell, 1970).
Estados Unidos ofreció una gran presencia mediática a estos investigadores como lo atestiguan algunos periódicos de la época (Kandell). Los casos abordados, las investigaciones sobre medidas labiales y las barras pintalabios, los métodos de recolección para validación forense y la ya clásica clasificación de estas huellas muestran a estos dos investigadores como el pináculo de la investigación queiloscópica.

\section{La huella en la fotografía}

En 1978, Kenneth J. Hoag reportó el caso de una huella labial en el dorso de una fotografía en poder de un soldado norteamericano comisionado en Europa. El individuo había sido acusado de violar a una joven, quien negaba conocerlo antes del incidente. El hombre alegaba haber sido su amante y que ella le denunciaba falsamente por despecho. En el dorso de la fotografía, junto a una dedicatoria cariñosa al acusado, podía verse una huella labial en pintalabios rojo briIlante. Aunque las pericias caligráficas no fueron determinantes, la fotografía fue enviada a la División de HueIlas Latentes del Comando de Investigación Criminal del Ejército de los Estados Unidos (USACIC) junto una impresión labial provista por la mujer. Las pericias queiloscópicas fueron realizadas mediante ampliaciones fotográficas y transparencias. Las comparaciones de ambas muestras resultaron en una identificación positiva; las sutiles diferencias en las formas fueron atribuidas a las variaciones de presión en la aplicación del pintalabios. La fiscalía retiró los cargos contra el acusado (Pertson, 1987).

\section{El robo al banco}

El prestigioso médico forense Dr. Michael Baden, relata para la cadena HBO otro singular suceso acontecido en California en 1978. Mientras los clientes de un pequeño banco realizaban sus operaciones, las cámaras de seguridad lograron registrar un suceso bastante particular: una mujer afroamericana, luego de amenazar con un arma de fuego a la dependiente y robar unos diez mil dólares, golpeó con su rostro una de las puertas de salida del banco al escapar, logró recuperarse y finalmente huir por otra puerta lateral. Advertidos por los testigos sobre este suceso (y comprobándolo con lo registrado en las cámaras), los detectives examinaron las puertas de cristal corroborando la presencia de una perfecta huella labial. Siguiendo procedimientos dactiloscópicos, los técnicos levantaron con cinta transparente el rastro fijándolo en fondo blanco. De la misma huella se reco- 
gió una segunda muestra revelada con polvos negros y ambos rastros fueron enviados a la división de huellas latentes del FBI. Aun cuando un anónimo denunció como autor a un hombre llamado Jonathan Jackson (quien ya poseía antecedentes de robo), los testigos alegaron haber visto una mujer y no a un hombre cometiendo el crimen. Esto no detuvo a los detectives, Jackson fue tomado en custodia. Los técnicos del FBI solicitaron al sospechoso que pintara sus labios con el mismo lápiz labial recuperado en la escena y que imprimiera su huella en una nueva muestra. Comparada con la obtenida en la puerta del banco, y siguiendo los mismos procedimientos utilizados en las dactilares, los detectives comprobaron que ambos patrones labiales eran idénticos. Jonathan Jackson fue encontrado culpable y condenado a 10 años de prisión (HBO, 1999) (Fig. 3).

\section{La carta indecente anónima}

John Berry, experto dactiloscopista del Cuerpo de Policía de Hertforshire (Inglaterra), reportó un interesante caso de identificación. La esposa de un ejecutivo preparaba las camisas de su marido para la limpieza cuando, al vaciar los bolsillos, descubrió un sobre azul fuertemente perfumado. Era una carta de alto contenido erótico, firmada con un beso en pintalabios. Cuando el hombre regresó a su hogar esa noche defendió enfáticamente no tener amante alguna y desconocer la identidad del remitente. Su esposa, creyéndole, insistió en que llevara la carta a la policía definiéndola como una "carta indecente anónima". EI sobre y la carta fueron procesados por Berry con Ninhidrina, pero al técnico le atrajo sobremanera la claridad de la huella labial. Como resultado, solicitó a

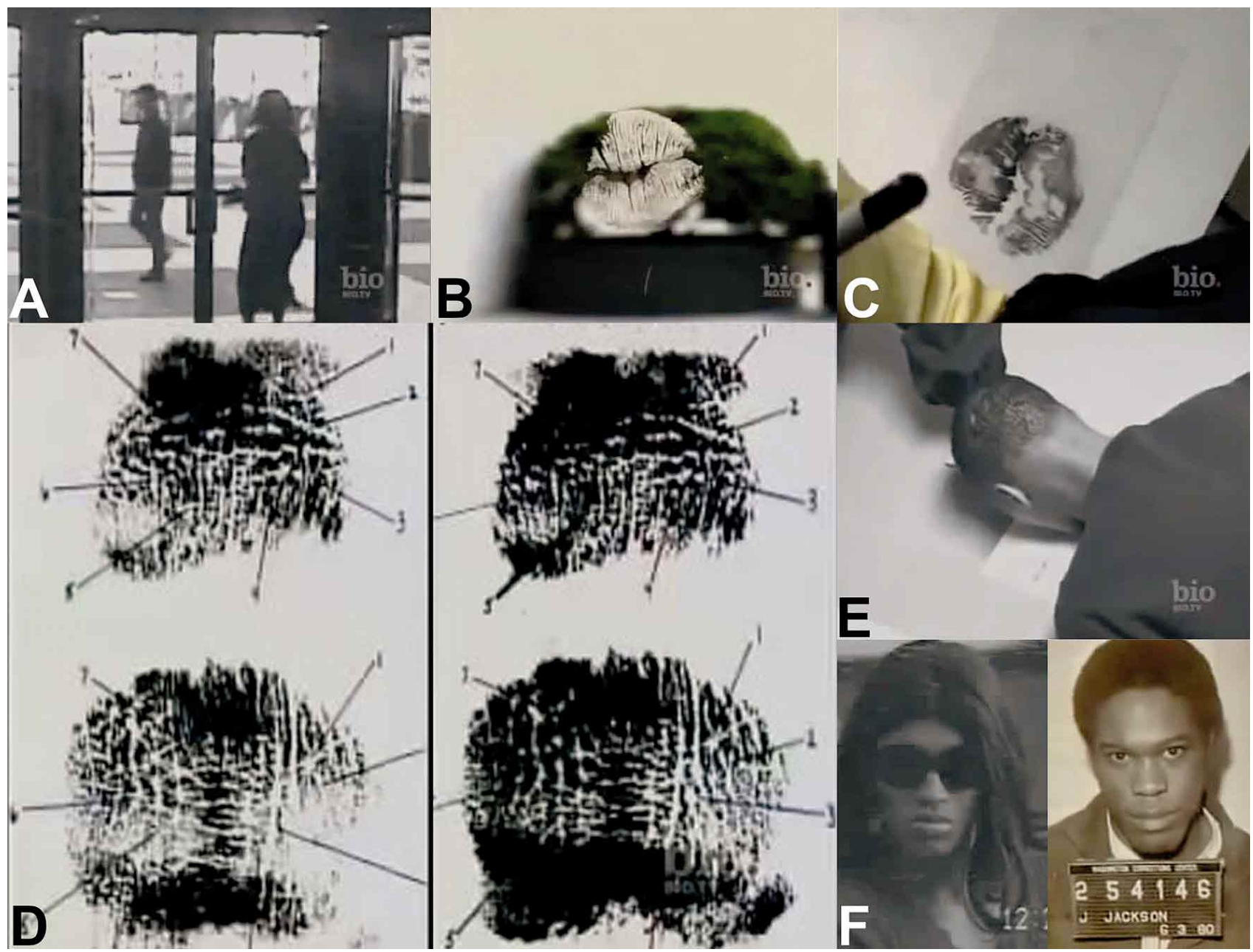

Fig. 3. A. El ladrón al momento de su golpe contra la puerta de vidrio durante el escape. B. Impronta labial revelada por técnicos del FBI. C. La misma huella clarificada en cinta y fondo blanco. D. Cotejo. E. Jonathan Jackson deja su huella indubitada para cotejo. F. Registro comparativo del rostro del ladrón -tomado del video de las cámaras de seguridad- y de J. Jackson (Adaptado del Documental de HBO Autopsy 6: Secrets of the Dead - The Telltale Imprint, 1999). 
la policía local que tomara impresiones labiales de todo el staff de la firma a pesar de que no existían precedentes o permisos algunos para tomar ese curso de acción. Sorpresivamente, todos cooperaron en unos pocos días, y quince juegos de huellas llegaron al laboratorio. Siguiendo un criterio algo arbitrario, Berry descartó las de los masculinos; media docena de huellas femeninas fueron examinadas permitiéndole rápidamente identificar la que coincidía con la del sobre: era la huella de la reacia y enojada secretaria del ejecutivo. Esta información fue enviada a los oficiales investigadores; la mujer admitió subsecuentemente haber enviado la carta (Pertson).

\section{Los casos polacos}

El Dr. Jerzy Kasprzak de la Escuela de la Escuela de Policía Militar en Minsk Mazowiecki (Polonia), publicaba en 1990 un artículo de referencia casi obligada, "Possibilities of Cheiloscopy". Según el autor, la primera investigación europea sobre las huellas labiales tuvo lugar en Hungría en 1961, a propósito del hallazgo de trazas labiales en una puerta de vidrio en la escena de un asesinato; el asesino habría empujado la puerta con la cabeza pues sus manos estaban manchadas de sangre. En Polonia específicamente, el interés por este tipo de evidencias comenzó en 1966 cuando, accidentalmente, una huella labial fue revelada en una ventana de vidrio en la escena de un robo. Aunque esta pericia fue desarrollada sin experiencia previa y concluyó exonerando al sospechoso (conclusión que el autor rebate aduciendo que nuevas técnicas podrían confirmar inequívocamente que las huellas pertenecían al acusado), sus resultados permitieron iniciar un intensivo trabajo de investigación al Departamento de Criminalística del Centro de Entrenamiento de la Policía Militar en Minsk Mazowiecki y más tarde a la División de Dactiloscopía del Departamento de Criminalística de Varsovia. Más de 7000 muestras de huellas labiales de 1500 voluntarios entre los 5 y los 60 años de todo el país fueron sistematizadas, analizadas y clasificadas, catalogando 23 tipos diferentes de propiedades individuales, y tres métodos queiloscópicos específicos para determinar patrones comunes, realizar fotomontajes y contornear las características labiales (Kasprzak, 1990, 2000). Sería recién en 1976 que una nueva pericia queiloscópica atraería la atención de la Policía Polaca: durante la inspección de una escena de robo en Milanówek, un técnico que buscaba huellas dactilares se topó con trazas labiales en una fotografía enmarcada detrás de la cual los propietarios guardaban su dinero. Luego del examen, corroboraron que la huella pertenecía a la hija de los propietarios (Kasprzak, 1990).

Las siguientes investigaciones en Polonia fueron desarrolladas a partir de 1985 , donde las metodologías empleadas fueron chequeadas primeramente en condiciones de laboratorio, probando su utilidad en la corte. En los años 1986 y 1987, se realizaron dos pericias queiloscópicas, ambas con opiniones positivas y categóricas. Similares resultados ofrecieron dos nuevos casos a mediados de abril de 1988. Kasprzak describe como muy interesante el del robo a un almacén en las vecindades de Ponsk en noviembre de 1987, donde fue encontrado un trozo de pastel con signos de mordedura. Luego de hacer los procedimientos para comparar las escayolas con las huellas de mordedura, los investigadores hallaron huellas labiales en la superficie del pastel, las que serían categóricas y mejoradoras de las mordeduras. El culpable pudo ser identificado más de tres meses después de ocurrido el suceso (Kasprzak, 1990).

\section{La huella en el vaso}

Uno de los más acérrimos defensores de la queiloscopía, el prominente Dr. Aggrawal (2004), relata un caso ocurrido en Japón durante una de sus visitas. Luego de violentar la entrada de una casa para robarla y casi a punto de escapar, el ladrón tomó un vaso de vidrio lleno de agua para calmar su sed. Aunque tenía guantes en sus manos, el individuo logró dejar una perfecta huella labial en el vaso. Durante la investigación, Aggrawal comparó esta huella con las de cinco sospechosos detenidos por la policía. Uno de ellos, un individuo de nombre Tsunachi, resultó positivamente identificado admitiendo más tarde su culpabilidad.

\section{Lavelle L. Davis}

El 18 de Diciembre de 1993, Patrick "Pall Mall" Fergusson era asesinado en un aparente intento de robo. La fiscalía presentó como única evidencia física una huella labial en un rollo de cinta adhesiva encontrada cerca del lugar del hecho, huella que coincidía con los labios de un individuo llamado Lavelle L. Davis. Leanne Gray, especialista en dactiloscopía del laboratorio forense de la Policía Estatal de Illinois y perito por la fiscalía, declaró haber revelado, fotografiado y preservado una huella de los labios superior e inferior en el lado adhesivo de la cinta. Gray tomó muestras de los labios de Davis, las comparó con la hallada en el lugar de los hechos y luego envió las fotografías a 
Steve McKasson, documentólogo del laboratorio científico forense de Southern Illinois en Carbondale. Unos meses después, y luego de realizar comparaciones adicionales, Gray y McKasson concluyeron que la huella pertenecía a Davis al haber encontrado al menos trece puntos de coincidencia (Fig. 4). Ambos peritos apoyaron sus afirmaciones en que la identificación por huellas dactilares constituía un método aceptado por la Policía del Estado de Illinois, por el FBI, y por la comunidad científica forense. El 25 de Julio de 1997, el jurado encontró culpable a Lavelle L. Davis con lo que fue condenado a 45 años de prisión, sentencia que fue reafirmada el 12 de Mayo de 1999 (Appellate Court of Illinois, Second District, 2007).

Hasta este punto, People vs. Davis constituye el caso más significativo admitiendo la identificación por huellas labiales en las cortes norteamericanas y ha sido referenciado en gran cantidad de publicaciones incluso hasta el año 2013 (Dineshshankar et al.). Sin embargo, la evolución real de este caso ha sido radicalmente diferente. Davis continuó apelando su condena y en Noviembre del 2005 la defensa presentó los testimonios de Andre Moenssens y Michael Sinke, peritos dactiloscopistas. Moenssens, profesor emérito de la Universidad de Missouri-Kansas y miembro de la
American Academy of Forensic Sciences (AAFS) opinó que la identificación por huellas labiales no estaba reconocida como ciencia aceptada, y que su investigación reveló la ausencia de estudios científicos validando su exactitud y confiabilidad, o prácticas metodológicas aceptadas dentro de la comunidad científica. Aseguró además la no existencia de sentencias judiciales afirmándolas como herramientas precisas y confiables explicando que no tenían publicaciones o programas formales de capacitación. Desconoció también si el FBI o alguno de sus miembros las respaldaba como método de identificación. El 7 de Marzo de 2006, la corte ingresó una orden de 22 páginas reconociendo la petición de Lavelle L. Davis y luego de cuestionar fuertemente a la defensa y a sus peritos (por haber permitido la primera condena), el 20 de noviembre del 2007, ordenó la inmediata liberación de Davis (Appellate Court of Illinois, Second District).

\section{DISCUSIÓN}

Rugoscopía y Queiloscopía representan, sin lugar a dudas, una batería de información transmitida desde hace más de 60 años donde la presencia de
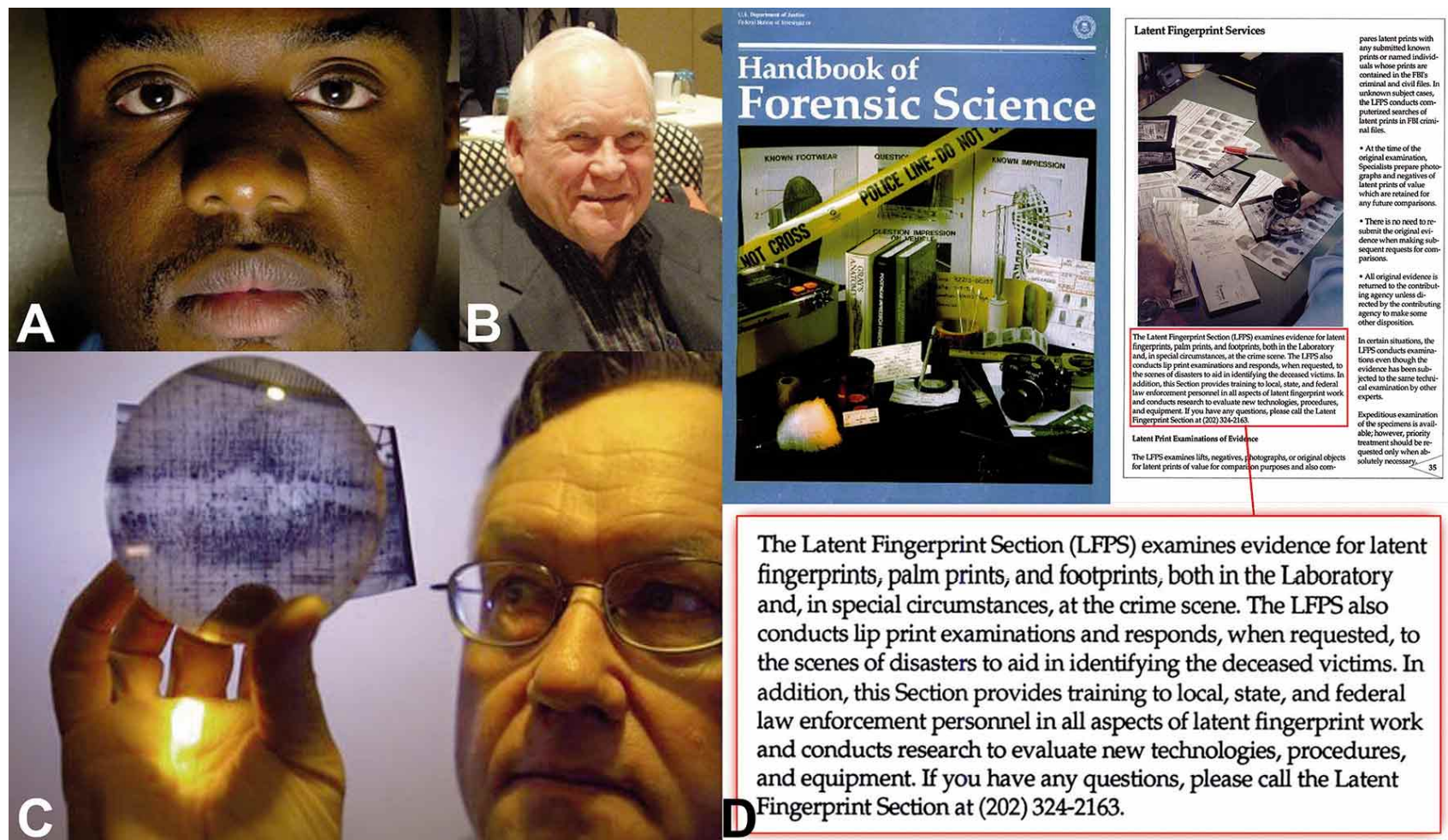

The Latent Fingerprint Section (LFPS) examines evidence for latent fingerprints, palm prints, and footprints, both in the Laboratory and, in special circumstances, at the crime scene. The LFPS also conducts lip print examinations and responds, when requested, to the scenes of disasters to aid in identifying the deceased victims. In addition, this Section provides training to local, state, and federal law enforcement personnel in all aspects of latent fingerprint work and conducts research to evaluate new technologies, procedures, and equipment. If you have any questions, please call the Latent Fingerprint Section at (202) 324-2163.

Fig. 4. A. Lavelle L. Davis. B. Andre Moenssens. C. Steve McKasson muestra la huella labial obtenida de la cinta adhesiva. D. Edición del año 1994 del Handbook of Forensic Science del FBI. A la derecha se ha resaltado el párrafo donde la Sección de Huellas Dactilares Latentes ofrece sus servicios de análisis de huellas labiales. 
casos paradigmáticos y menciones célebres han pretendido justificar su inclusión en la investigación criminalística y medico-legal. En el caso de la identificación por rugas palatinas, si bien existen numerosas revisiones e investigaciones originales, los únicos tres casos detectados expusieron una situación de no menor importancia: solo el azar de contar con prótesis conocidas como pertenecientes al fallecido permitiría realizar las identificaciones (se profundiza todavía más el conflicto de contar con la necesaria información AM para un cotejo positivo). Si bien es cierto, la eventual posibilidad de identificar odontológicamente a un individuo edéntulo resulta sumamente atractiva para el forense, el revelado de las rugas desde las prótesis y la posibilidad de distorsión originadas por procesos distróficos, reabsortivos o inflamatorios en los portadores, exponen serios dilemas técnicos y procedimentales. Esta revisión detectó sólo tres investigaciones originales replicando esta particular maniobra en una situación controlada, uno de ellos informando solo un $79 \%$ de certeza en los cotejos (Jacob \& Shalla, 1987). Pérdidas óseas, rugas poco demarcadas, inconsistencias entre observadores, técnicas defectuosas de impresión o vaciado, fueron las explicaciones dadas por los autores para justificar los errores informados. Alexander et al. (1998) aseveraron que la comparación del patrón de rugas de una prótesis con las de la boca del fallecido puede corroborar pero nunca ser la única evidencia para una identificación positiva, defendiendo que nunca van a poseer el mismo valor que un código o número identificatorio grabado en la base protésica. Entendemos que la investigación rugoscópica debería sustentarse en las alternativas ofrecidas por las rehabilitaciones protésicas, pero aún más en la concepción técnica del procedimiento. Coincidimos con Segelnik \& Goldstein (2005) en que los hallazgos de la literatura al respecto son conflictivos e insuficientes y que la mayoría de los estudios han sido realizados con un escaso número de muestras.

\section{FBI y Huellas Labiales}

Con respecto a las huellas labiales, aunque los reportes de casos abundan y los estudios poblacionales apoyados en la clasificación de Suzuki \& Tsuchihashi parecen constituir foco en la literatura proveniente en su gran mayoría de India (Dineshshankar et al.), el paradigma angloparlante continúa escéptico al respecto. Volviendo al caso de Lavelle L. Davis y reafirmando los dichos de Andre Moenssens (quien reconocía desconocer algún tipo de respaldo del FBI hacia las técnicas queiloscópicas), determinante para la liberación de Davis fue la nota emanada del equipo de rastros de ese organismo federal norteamericano: "(nuestro laboratorio) no ha llevado a cabo ningún estudio validando la identificación mediante huellas labiales y no realizará análisis alguno de este tipo de rastros" (Appellate Court of Illinois, Second District). Ann Todd, portavoz de la entidad en Quantico, aseguró a los medios de prensa: "hasta este momento, no han sido validadas las comparaciones de huellas labiales" (McRoberts et al., 2004). El periodismo recibió esta información con mucha plana amarilla (The Chicago Tribune tituló su nota con un ácido "Forensic Under the Microscope. Unproven Techniques Sway Courts, Erode Justice") (McRoberts et al.). La comunidad científica de todo el mundo se mantuvo en inusitado (y peligroso) silencio.

Sería oportuno aquí remontarse al año 1992, cuando Mary Lee Schnuth, una profesora asociada de la Old Dominion University en Norfolk (Estados Unidos), aseveraba que "los estudios realizados con huellas labiales refuerzan su utilización en la resolución de crímenes" y que "muchas entidades judiciales desconocen su utilidad cuando intentan identificar sospechosos, perdiendo con ello importante evidencia". Y concluía: "Las fuerzas del orden deberían comenzar a considerar los análisis de huellas labiales como otra herramienta a utilizar para la resolución de crímenes". El artículo se titulaba "Focus on Forensics; advantages of lip print analysis in criminal investigations" y la publicación correspondía al número de noviembre de ese año del FBI Law Enforcement Bulletin (LEB) (Schnuth, 1992). Según el sitio oficial de la entidad, el LEB ha sido desde 1935 el medio oficial de comunicación y transmisión de información e investigación científica de la fuerza, con copias impresas y digitales enviadas a más de 100 países en el mundo. Debe recordarse que ya en 1979, los laboratorios de rastros del FBI habían contribuido a la captura de Jonathan Jackson luego de dejar este su huella labial en la puerta del banco (HBO). La edición 1994 del Handbook of Forensic Science (FBI, 1994) menciona en su página 35 que sus laboratorios ofrecían entre sus servicios los análisis queiloscópicos (Fig. 4). Ball (2002) del Center for Forensic Science en la Universidad de Western Australia, en otro de los artículos más citados por la literatura especializada, afirmaba en el año 2002 que la Sección de Rastros del FBI en Washington DC realizaba periódicamente talleres focalizados en los análisis de huellas labiales.

¿Qué sucedió entonces ese año 2007 con la queiloscopía -ciencia identificatoria reconocida y 
ponderada por el FBI solo algunos años atrás? La absolución de Lavelle L. Davis coincidía con la edición de ese año -la última- del Handbook of Forensic Services: los análisis de huellas labiales ya no figuraban entre sus servicios. Debe contextualizarse este cambio; el sistema judicial norteamericano no pasaba un buen momento en lo que refiere a pericias y peritos. Los finales del siglo XX e inicios del XXI habían puesto en escena a la genética forense, The Innocence Project, las normas Daubert, la gran cantidad de condenas injustas con foco en la Odontología Forense (Fonseca et al., 2013) y más todavía el enorme el fiasco sufrido por el FBI con la identificación errónea del supuesto culpable del atentado en Madrid en 2004, razones que cuestionaban severamente la credibilidad de sus laboratorios (McRoberts et al.; Saks \& Koehler, 2005). Kelly \& Wearne (1988), en el libro nominado al Pulitzer Tainting Evidence: Inside the Scandals at the FBI Crime Lab, mencionan que los directores de los laboratorios del FBI "...conocían la real significación de romper algunas de las más fundamentales reglas de la práctica científica. Han estado en connivencia tanto con la incompetencia de los examinadores para evitar cualquier vergüenza posible a la institución, como con lo tendencioso de los exámenes porque estos aseguraban "resultados". No es de extrañar entonces la retracción del FBI y de la mayoría de las instituciones reconocidas ante esta presión. La AAFS, en voz de su entonces Presidente Ronald Singer, mencionaba: "huellas de orejas, labios, nariz o codos recaen todas en la misma categoría: no verificadas ni validadas". Luego de la absolución de Davis, Steve McKasson defendió su pericia a la prensa mostrando en un computador los trece puntos de semejanzas identificados en su trabajo: "Aún no encuentro otra explicación porque allí estaban y para mí estaban bien. Esas dos huellas provenían de la misma persona. No tengo ninguna duda". Respecto a la negación expresa del FBI, afirmó: "me molesta la flojera de algunos de ellos. Solamente les preocupa el que vayan a ser atacados" (McRoberts et al.).

Ya se ha visto que el informe del año 2009 de la National Academy of Sciences (NAS) pretendió exponer de manera definitiva cuáles eran hasta ese momento las vulnerabilidades y posibles soluciones en determinados tópicos forenses (Fonseca et al.). Respecto a las huellas labiales, el informe aceptó que siendo un tipo de rastro infrecuente, los problemas para homologarlos como evidencia identificatoria son aún mayores; citando una encuesta realizada por investigadores finlandeses, NAS menciona que los mínimos estudios en huellas labiales (14 laboratorios informaron haber realizado un total de 21 análisis queiloscópicos anuales, con una media por laboratorio de 2 casos por año) resultan escasos para afirmar suficiencia en la variabilidad de las huellas (Committee on Identifying the Needs of the Forensic Science Community et al., 2009). Creemos que esta afirmación es circunstancial y poco objetiva... ¿hasta qué punto los laboratorios están capacitados para reconocer o procesar este tipo de evidencia? Pertson, de la Sección Identificación de la Real Policía Montada Canadiense en Yorkton (Canadá), admite haber encontrado en 1984 una impresión parcial de labios humanos en una ventana forzada que no consideró como potencialmente utilizable como evidencia. Petersen (2009), siendo investigador en la División de Investigación Criminal de la Marina de los Estados Unidos, reconoce no haber procesado una huella labial en una ventana en un caso de voyeurismo, pues sus superiores no conocían cómo debían hacerlo. ¿Habrá llegado años después a oídos de Petersen el caso de Robert Neal Smith en Newport, sentenciado a 5 meses de prisión precisamente por voyeur, también gracias a una huella labial dejada en una ventana de vidrio, esta vez procesada y comparada por el Laboratorio de Criminalística de la Policía estatal en Richmond? (Archibald et al., 2011).

Es clara la afirmación de Kasprzak (1990): “El empleo práctico de las huellas labiales en el trabajo de detección, demuestra que este tipo de rastros contiene una enorme cantidad de preciosa información que puede ser utilizada en la reconstrucción del evento estableciendo versiones, comprobándolas e identificando a los sospechosos". Y concluye: "La utilización eficiente de las huellas labiales depende de un alto nivel de habilidad de los miembros de las fuerzas del orden. Los problemas involucrados en la queiloscopía son relativamente poco conocidos entre nuestros criminalistas debido a que las huellas labiales han sido usadas sólo esporádicamente hasta ahora, a pesar de su frecuente aparición en la escena del crimen. La única solución posible es colocar a la queiloscopía dentro del ámbito de trabajo de los criminalistas, junto a la dactiloscopía y otros medios de identificación personal y presentarla dentro del plan de capacitación de los investigadores criminales". Muy positiva pareció ser esta nueva competencia del Departamento de Huellas Dactilares del Laboratorio Central Forense de la Policía en Varsovia: durante los años 1985 a 1997, el laboratorio utilizó técnicas queiloscópicas en 85 casos incluyendo 65 robos, 15 homicidios y 5 asaltos. En 34 de ellos 
las identificaciones fueron positivas (Kasprzak, 2000). Son prometedoras las actividades de capacitación brindadas a cuerpos de Policía Judicial en Argentina (Bonfigli et al., 2010) o las puestas a prueba en medios controlados de diferentes reactivos y soportes en el Laboratorio de Huellas de la Escuela de Investigaciones Policiales de la Policía de Investigaciones en Chile (Ramos et al., 2013).

Esto pone en evidencia otro elemento necesario de gran relevancia: la relación equilibrada y sana entre científicos y técnicos. Mientras que la Real Academia Española define al investigador como quien "amplía el conocimiento científico sin perseguir, en principio, ninguna aplicación práctica", el investigador criminal funda su trabajo precisamente en la aplicación práctica de estos preceptos: la búsqueda concreta y técnica de evidencias físicas. Esta dicotomía no es solo aparente; más allá de lo singular de un conocimiento en un contexto científico, sólo la aplicación fáctica de él en un caso, siguiendo los estándares técnicos en respeto absoluto de las normas procesales va a permitir su aceptación en juicio. El perito debe ser un conocedor profundo de las herramientas que utiliza y de su viabilidad jurídica. Burns (1981) luego de detallar métodos y equipamiento para la fijación, fotografía y comparación de huellas labiales, recomendaba que antes de registrarlas se realicen las consultas pertinentes con las autoridades judiciales locales para evaluar qué tanto pueden verse afectadas como evidencia.

Aunque los reportes de casos suelen ser considerados "inferiores" por su naturaleza anecdótica (incluso rechazados por algunas revistas prestigiosas), en ciencias forenses éstos logran exponer áreas, conductas y procedimientos imposibles de reproducir experimentalmente, representando una excelente oportunidad de aprendizaje y de revisión permanente de cada uno de los factores que los afectaron en su desarrollo (Madea; Fonseca et al.). Si bien rugoscopía y queiloscopía han supuesto seductoras líneas de presentación de la odontología forense, lo cierto es que la notoria ausencia de reportes de casos en revistas arbitradas y validadas, y la virtual (o real) inaccesibilidad de los pocos publicados han determinado rutinas "copy-paste" donde citas de citas han engrosado los marcos referenciales socavando precisamente lo que pretendían cimentar. Steven Jones, primer abogado defensor de Timothy McVeigh en el caso de la bomba en Oklahoma, alega que "básicamente, lo que tenemos es una especie de tradición oral, como el inglés medieval o Beda el Venerable en vez de un manual de protocolo regular científico" (Kelly \& Wearne). Referenciar sin corroborar de manera directa es tan negligente como utilizar un procedimiento forense fuera de los protocolos aceptados por la comunidad científica. Es preferible una cita ausente que una cita errónea o fuera de contexto.

Sin dudas, los análisis rugoscópicos y queiloscópicos necesitan de investigación y de un sistemático diseño experimental no solo con perfilaciones antropológicas sino también con la reproducción de situaciones reales de cotejo. Problemas en los revenidos de las rugas o en sus falsificaciones; las deformaciones, arrastres, parcialidad o sobreimpresión de las huellas labiales y en ambos casos la presencia de patologías, variaciones postmortem, el poder contar con una base de datos antemortem/indubitada (Caldas et al.) o el análisis de los códigos procesales para la inclusión efectiva de estos rastros como evidencia jurídica, han originado hipotéticas soluciones pero escasas puestas a prueba en campo (Bonfigli et al.). El paradigma actual continúa reafirmando los análisis cuantitativos por sobre los cualitativos, en clara previsión de las observaciones subjetivas y sus posibilidades de sesgo. En 2007, durante el $18^{\circ}$ Simposio Internacional de Identificación Humana, Tony Tambasco presentó el caso de un robo a mano armada en el que el perpetrador, al abandonar la escena, dejó una huella labial en una puerta de vidrio de la cual el Laboratorio de la Policía de Mansfield extrajo el ADN con el que sería culpado y condenado (Archibald et al.). Son bien conocidas las limitaciones de las pruebas genéticas pero todo parece indicar que los tribunales continuarán ratificando su seguridad y verificabilidad. Quizás es tiempo de reformular los mismos principios de la Odontología Forense y de sus peritos para cimentar sus fortalezas y trabajar en sus debilidades. Los análisis objetivos de los casos reportados y el ser partícipes con los propios de una rutina de publicación validada (algo desafortunadamente inusual en la literatura de habla hispana), pueden ser excelentes puntos de partida.

\section{AGRADECIMIENTOS}

A la Dra. Helen James, Director of the Forensic Odontology Unit, University of Adelaide, Australia, y al Sr. Frédéric Gaudreau, Team Leader of the Acquisitions and Collections Management, Canadian Police College Library, Royal Canadian Mounted Police, Ottawa, Canada. 
FONSECA, G. M.; CANTÍN, M. \& LUCENA, J. Forensic Dentistry III: Palatal rugae and lip prints in forensic identification. Int. J. Odontostomat., 8(1):29-40, 2014.

ABSTRACT: Even though there exist more or less frequent methods of human identification in use, all of them are supported in the unequivocal, scientific and reliable comparison of known information with the obtained one of the remains or of the prints to processing. Between these methods, and from a dental forensic point of view, the lip prints, the palatal rugae, and their negatives or prints have been admitted as morphologic patterns, which would allow identifying a person in specific circumstances. These elements have been studied comparatively as well as independently trying to detect individual and demographical characteristics that allow to reinforce them as significant tools: it was informed that the Cheiloscopy (the study of the lip prints) as well as the Rugoscopy (the study of palatal rugae) both define unique traits for every individual; therefore, they are useful reasonably for a forensic identification.Since the forensic case reports may help to identify changes in criminal behaviours, in the morphologic typical patterns or in the way of presenting the expert evidence, a review of case reports with utilization of rugoscopical and cheiloscopical evidence is presented, and their projections toward the scientific forensic community are discussed.

KEY WORDS: forensic dentistry, rugoscopy, cheiloscopy, case reports

\section{REFERENCIAS BIBLIOGRÁFICAS}

Aggrawal, A. The importance of lip prints. Mystery magazine web. 2004. Disponible en: http:// lifeloom.com/II2Aggrawal.htm

Alexander, P. M.; Taylor, J. A.; Szuster, F. S.; Brown, K. A. An assessment of attitudes to, and extent of, the practice of denture marking in South Australia. Aust. Dent. J., 43(5):337-41, 1998.

Aparicio Castellanos, D. C.; Henríquez Higuera, L. F.; Hurtado Avella, A. M.; Pedraza Gutiérrez, A. P.; Casas Martínez, J. A. Identificación positiva por medio del uso de la rugoscopía en un Municipio de Cundinamarca (Colombia): Reporte de caso. Acta Odontol. Venez., 45(3):e1-6, 2007.

Appellate Court of Illinois, Second District. The People of the State of Illinois, Plaintiff-Appellant, v. Lavelle L. Davis, Defendant-Appellee. No. 2-06-0319, November 20, 2007.

Archibald, N.; Cullen, L. \& Bikker, J. Identification from soft tissues. En: Black, S. \& Ferguson, E. (Eds.). Forensic Anthropology 2000 to 2010. Boca Raton, CRC Press, 2011.

Ball, J. The current status of lip prints and their use for identification. J. Forensic Odontostomatol., 20(2):43-6, 2002.

Bonfigli, E. A.; Trujillo, G.; Cantín, M.; Fonseca, G. M. Procedimientos y aprendizaje significativo en la investigación criminal. Presentación de dos experien- cias de capacitación interdisciplinaria. FOPJ, 1(2):14-9, 2010.

Burns, R. W. A Kiss for the Prosecution. Detective, 8(2):24-7, 1981.

Caldas, I. M.; Magalhães, T. \& Afonso, A. Establishing identity using cheiloscopy and palatoscopy. Forensic Sci. Int., 165(1):1-9, 2007.

Committee on Identifying the Needs of the Forensic Science Community; Committee on Science, Technology \& Law Policy and Global Affairs, Committee on Applied and Theoretical Statistics, Division on Engineering and Physical Sciences. National Research Council of the National Academies. Strengthening Forensic Science in the United States: A Path Forward. Washington DC, The National Academies Press, 2009.

Dineshshankar J.; Ganapathi, N.; Yoithapprabhunath T. R.; Maheswaran, T.; Kumar, M. S. \& Aravindhan, R. Lip prints: Role in forensic odontology. J. Pharm. Bioallied Sci. 5(Suppl. 1):S95-7, 2013.

FBI. Handbook of forensic science. Washington DC, US Government Printing Office, 1994.

Fonseca, G. M.; Briemm-Stamm, A. D.; Cantín, M.; Lucena, J. \& Bentkovski, A. Odontología Forense I: Las Huellas de Mordedura. Int. J. Odontostomat., 7(1):149-57, 2013. 
HBO. Autopsy 6: Secrets of theDead - The Telltale Imprint. Video, Octubre, 1999.

Jacob, R. F. K. \& Shalla, C. L. Postmortem identification of the edentulous deceased: Denture Tissue Surface Anatomy. J. Forensic Sci., 32(3):698-702, 1987.

Jones, L. V. Scientific Investigation and Physical Evidence. A Handbook for Investigators. Springfield, Charles C. Thomas, 1959.

Kandell, J. L. Lip prints: New aid in fight against crime. Boca Raton News, 26 de Noviembre, 1970.

Kasprzak, J. Possibilities of Cheiloscopy. Forensic Sci. Int., 46(1-2):145-51, 1990.

Kasprzak, J. Cheiloscopy. En: Siegel, J.; Knupfer, G. \& Saukko, P. (Eds.). Encyclopedia of Forensic Sciences. Three-Volume Set, 1-3. East Lansing, Academic Press, 2000.

Kelly, J. F. \& Wearne, P. K. Tainting Evidence: Inside the Scandals at the FBI Crime Lab. New York, Free Press, 1998.

Madea, B. Case histories in forensic medicine. Forensic Sci. Int., 17;165(2-3):111-4, 2007.

McRoberts, F.; Mills, S. \& Possley, M. Forensic Under the Microscope. UnprovenTechniques Sway Courts, Erode Justice. The Print, 20(6):1-7, 2004.

Nambiar, P.; Jalil, N. \& Singh, B. The dental identification of victims of an aircraft accident in Malaysia. Int. Dent. J., 47(1):9-15, 1997.

Pertson, W. J. Ear, lip and nose prints: another means of identification. R.C.M.P. Gazette, 49(9):12-5, 1987.

Petersen, L. C. Lip prints. A thesis submitted to the National University, La Jolla, CA, USA, March 2006. Anil Aggrawal's Internet Journal of Forensic Medicine and Toxicology, 10(1), 2009. Disponible en: http://www.anilaggrawal.com/ij/vol_010_no_001/ others/thesis/2/petersen_thesis.doc

Ramos, C.; Meyer, J.; Castro, J. \& Espinoza, B. La queiloscopía como sistema de identificación complementaria en el área de investigación de homicidios por parte de los oficiales y peritos del Labora- torio de Criminalística de la PDI. Tesis Academia Superior de Estudios Policiales, Policía de Investigaciones de Chile, Santiago, 2013.

Saks, M. J. \& Koehler, J. J. The coming paradigm shift in forensic identification science. Science, 309(5736):892-5, 2005.

Schnuth, M. L. Focus on Forensics; advantages of lip print analysis in criminal investigations. The FBI Law Enforcement Bulletin. November 1, 1992. Disponible en http://www.thefreelibrary.com/Lip+prints.a013690176

Segelnick, S. L. \& Goldstein, L. Forensic application of palatal rugae in dental identification. Forensic Examiner, 14:44-7, 2005.

Snyder, L. Homicide Investigation: practical information for coroners, police officers, and other investigators. Springfield, Charles C. Thomas, 1950.

Suzuki, K. \& Tsuchihashi, Y. Two criminal cases of lip print. Forensic Sci, 5(2):171, 1975.

Thomas, C. J. \& van Wyk, C. W. The Palatal Rugae in an Identification. J. Forensic Odontostomatol., 6(1):21-7, 1988.

Dirección para Correspondencia:

Gabriel M. Fonseca, D.D.S., Ph.D.

Mariano Moreno 937, Cordoba

Zip code: X5000MRS

ARGENTINA

Email: gabriel_fonseca@argentina.com

Recibido : 17-11-2013

Aceptado: 12-01-2014 